\title{
Survival of patients with synchronous brain metastases: an epidemiological study in southeastern Michigan
}

\author{
Ajith J. Thomas, M.D., Jack P. Rock, M.D., Christine C. Johnson, Ph.D., \\ Linda Weiss, Ph.D., Gordon Jacobsen, M.S., and Mark L. Rosenblum, M.D. \\ Departments of Neurosurgery and Cancer Epidemiology Prevention and Control, Josephine Ford \\ Cancer Center Henry Ford Health System, Barbara Karmanos Cancer Center, Detroit, Michigan; \\ and Biostatistics and Research Epidemiology, Henry Ford Health System, Detroit, Michigan
}

\begin{abstract}
Object. It has been suggested that synchronous brain metastases (that is, those occurring within 2 months of primary cancer diagnosis) are associated with a shorter survival time compared with metachronous lesions (those occurring greater than 2 months after primary cancer diagnosis). In this study the authors used data obtained from the National Cancer Institute's Surveillance, Epidemiology, and End Results Program to determine the incidence of synchronous brain metastases and length of survival of patients in a defined population of southeastern Michigan residents.

Methods. Data obtained in 2682 patients with synchronous brain metastases treated from 1973 to 1995 were reviewed. Study criteria included patients in whom at least one brain metastasis was diagnosed within 2 months of the diagnosis of primary cancer and those with an unknown primary source. The incidence per 100,000 increased fivefold, from 0.69 in 1973 to 3.83 in 1995 . The most frequent site for the primary cancer was the lung (75.4\%). The second largest group $(10.7 \%$ ) consisted of patients in whom the primary site was unknown. The median survival length was 3.3 months. There was no significant difference in the median survival in patients with primary lung/bronchus and those with an unknown primary site (3.2 months and 3.4 months, respectively).

Conclusions. Patients who present with synchronous lesions have a poor prognosis, and the predominant cause of death, in greater than $90 \%$ of cases, is related to systemic disease; however, despite poor median survival lengths, certain patients will experience prolonged survival.
\end{abstract}

KEY WORDS • epidemiology • synchronous • brain metastasis • survival

Brain metastases are being seen with increasing frequency in hospital-based studies and surgical series.,11 Since 1972, no population-based studies have been conducted in the United States in which the incidence of brain metastases is addressed. ${ }^{19} \mathrm{An}$ increase in the ratio of brain metastases to primary tumors in hospital-based series and surgical series has been noted. This could be related to a number of factors including improved treatment of primary cancer and systemic metastases resulting in an increase in survival times that, in turn, may provide time for cells sequestered in a protective "sanctuary" behind the bloodbrain barrier to multiply and become symptomatic. ${ }^{13,17,18}$ In addition, with an aging population, more people are at risk for developing primary systemic cancer, and as a result, the number of brain metastases will also increase. Finally, a greater awareness on the part of physicians of the favorable brain metastases-related management strategies coupled with improved neuroimaging capability have also contributed to the increased incidence of diagnosed brain metastases. ${ }^{3,4,7,15}$

Abbreviations used in this paper: $\mathrm{CT}=$ computerized tomography; SEER = Surveillance, Epidemiology, and End Results.
There are only three population-based published studies on the incidence of metastatic tumors of the brain. The only study recorded in the United States was performed at the Mayo Clinic and published in 1972. The other studies were conducted in Scandinavian countries, ${ }^{8,9}$ the last being reported in 1984. Therefore, all of these studies involved people of predominantly Scandinavian origin as opposed to the more diverse ethnic distribution usually encountered in North American clinical practice. These studies also antedate the development of more sophisticated methods of neurodiagnostic imaging.

In conjunction with the apparent increase in the incidence of brain metastases, there has been a willingness to treat such lesions more aggressively. ${ }^{6}$ For the purposes of developing clinical trials and determining allocation of resources, studies designed to determine the incidence of brain metastases and patient survival in a defined population have become important. In this project, we focus on the incidence of synchronous brain metastases and the survival of patients harboring these lesions.

\section{CLINICAL MATERIAL AND METHODS}

The SEER Program of the United States National Can- 
TABLE 1

Patients with brain metastasis detected within 2 months of primary diagnosis*

\begin{tabular}{ccc}
\hline \hline $\begin{array}{c}\text { Years of } \\
\text { Observation }\end{array}$ & Year & Incidence \\
\hline 1 & 1973 & 0.690 \\
2 & 1974 & 0.869 \\
3 & 1975 & 1.252 \\
4 & 1976 & 1.252 \\
5 & 1977 & 1.380 \\
6 & 1978 & 2.045 \\
7 & 1979 & 2.045 \\
8 & 1980 & 2.556 \\
9 & 1981 & 3.067 \\
10 & 1982 & 2.760 \\
11 & 1983 & 3.271 \\
12 & 1984 & 3.246 \\
13 & 1985 & 3.731 \\
14 & 1986 & 3.271 \\
15 & 1987 & 4.549 \\
16 & 1988 & 4.396 \\
17 & 1989 & 3.680 \\
18 & 1990 & 4.089 \\
19 & 1991 & 4.115 \\
20 & 1992 & 4.192 \\
21 & 1993 & 3.987 \\
22 & 1994 & 4.268 \\
23 & 1995 & 3.834 \\
\hline
\end{tabular}

* Incidence per 100,000 residents of southeastern Michigan tricounty region.

cer Institute has been collecting information on cancer since 1973 from population-based cancer registries in a selected number of areas in the United States. The metropolitan areas included in the SEER registry are San Francisco-Oakland, Atlanta, Detroit, and Seattle-Puget Sound, and the states are Connecticut, Hawaii, Iowa, New Mexico, Utah, and New Jersey. Puerto Rico is also included. We used this registry to identify patients with metastatic brain tumors.

The project consisted of a retrospective assessment of new cases in which at least one synchronous brain metastasis was identified between 1973 and 1995 in the tricounty area of southeastern Michigan. We defined "synchronous" brain metastases as those lesions in the brain that were detected within 2 months of the primary diagnosis of related cancer and those with an unknown primary site. In the SEER program patients with metastatic lesions are not aggressively recruited or followed per se, and therefore, the maximum yield of cases of metastases occurs when lesions are discovered during the first 2 months after the primary cancer is diagnosed. Therefore, even though the SEER program provides the best record of population-based data, it is most likely an underestimation of the actual incidence of disease. For the purposes of this investigation, newly diagnosed metastatic brain lesions in patients in whom the primary cancer site was unknown were also considered as synchronous (288 cases). Because the time interval between diagnosis and establishment of brain metastases in patients with unknown primary cancers presenting with brain metastases might not be similar to those in whom the time interval is known, we have stratified the data to allow for separation. When
TABLE 2

Primary site for metastatic lesions of the brain

\begin{tabular}{lc}
\hline \hline \multicolumn{1}{c}{ Site } & Frequency $(\%)$ \\
\hline esophagus & $10(0.4)$ \\
stomach & $10(0.4)$ \\
colon & $39(1.5)$ \\
nasal cavity & $21(0.8)$ \\
lung \& bronchus & $2023(75.4)$ \\
skin melanoma & $25(0.9)$ \\
breast & $60(2.2)$ \\
prostate & $10(0.4)$ \\
kidney & $58(2.2)$ \\
lymphoma & $17(0.6)$ \\
unknown primary & $288(10.7)$ \\
other & $121(4.5)$ \\
\hline
\end{tabular}

a patient harbored multiple primary lesions, the primary cancer most closely related temporally to the detection of the metastatic lesion was considered to be the primary cancer that had metastasized.

The SEER Program obtains its data by creating abstracts of the medical records obtained on patients from participating hospitals. The SEER database codes information on the presence of metastases but not on the type of evidence that substantiates that diagnosis. Multiple types of evidence may be used to document the diagnosis, including CT scanning, magnetic resonance imaging, or other sources of information (for example, by a notation made by the treating physician, or from autopsy/biopsy results).

The SEER Program has maintained stringent guidelines and quality control over data since its year of inception; however, the quality of medical records obtained from participating hospitals may have improved over the 1973 to 1995 time period.

We used census data to define the demographic characteristics of the population in the southeastern Michigan tricounty area and then used these data to evaluate the incidence of synchronous brain metastases and the survival of these patients.

Registry data recorded over the 23-year period included age, sex, marital status, previously diagnosed forms of cancer and their primary sites, race, histological appearance, diagnostic confirmation of the primary cancer, and survival data including date of death, whether an autopsy was performed, and the cause of death listed on the death certificate. Cases were tabulated on an annual basis from January 1973 to December 1995. The population of interest is dynamic, and thus census data collected from different time periods was used in the analysis. The total population for the region of interest in 1980 was 4,044,284 and this number had decreased to $3,912,679$ by 1990 .

\section{RESULTS}

Two thousand six hundred eighty-two patients met the criteria for having synchronous brain metastases from 1973 to 1995 . The incidence of synchronous brain metastases increased from 0.69 per 100,000 population to 3.83 per 100,000 (Table 1). Lung cancer was the most common 
TABLE 3

Histological appearance of primary tumors

\begin{tabular}{lc}
\hline \hline Histological Appearance & Frequency $(\%)$ \\
\hline well differentiated & $32(1.2)$ \\
moderately differentiated & $170(6.3)$ \\
poorly differentiated & $819(30.5)$ \\
undifferentiated & $443(16.5)$ \\
(grade) undetermined & $1209(45.1)$ \\
others & $9(0.3)$ \\
\hline
\end{tabular}

primary site, accounting for $75.4 \%$ of the metastatic lesions, whereas brain metastases associated with an unknown primary cancer accounted for $10.7 \%$. Breast, colon, and kidney cancer were the other primary locations most commonly encountered (Table 2 ).

Of the 2682 patients, $58.5 \%$ were married, $61.2 \%$ were men, and $38.7 \%$ were women. African Americans represented $25.8 \%$ of the population and Caucasians $74.2 \%$. No in situ primary tumors were observed (Table 3). Although occasional long-term survival was observed, the overall median survival length was only 3.2 months after diagnosis of the brain lesion (Figs. 1 and 2 and Table 4). The cause of death was listed as the brain lesion in only $2.5 \%$ of the cases.

\section{DISCUSSION}

Hospital-based studies, especially referral hospitals, provide us with information about selected groups of patients, and studies on survival data associated with surgical series may reflect an especially strong selection bias for patients with better survival outcomes. Populationbased data tend to eliminate this referral bias and represent our best means of analyzing a particular disease for largearea demographic variations. Since 1973, the population of the southeastern Michigan tricounty area has been part of the SEER Program, one of the world's most well-documented and comprehensive cancer registry programs.

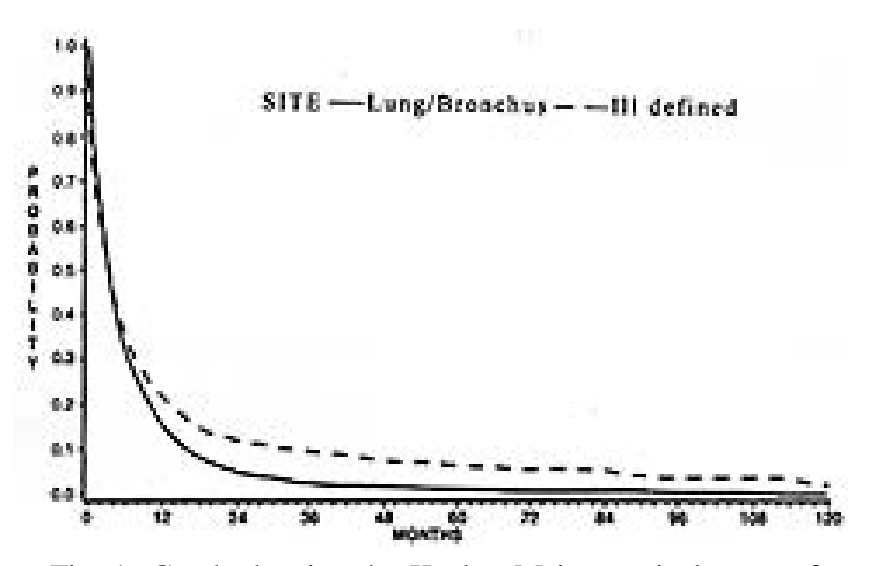

Fig. 1. Graph showing the Kaplan-Meier survival curves for patients with brain metastases demonstrated within 2 months of primary diagnosis of bronchogenic carcinoma or unknown primary site.

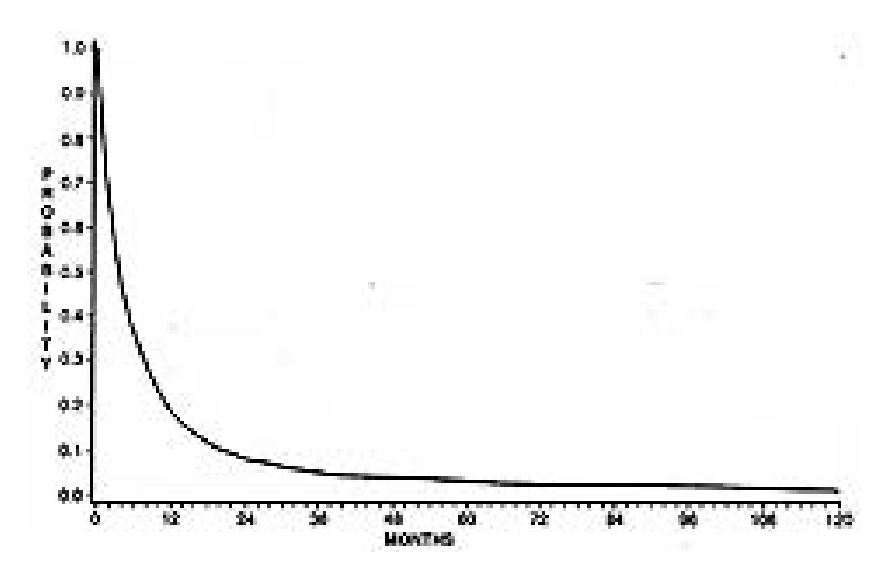

Fig. 2. Graph showing the Kaplan-Meier survival curve for patients with brain metastasis detected within 2 months of primary cancer diagnosis.

Analysis of this database demonstrated that the incidence of cancer with synchronous metastatic lesions to the brain increased markedly from 1973 to 1987 and has held constant since 1987. In part, the explanation for this increase lies in the earlier and more accurate diagnosis resulting directly from improved neurodiagnostic imaging modalities. From indirect brain imaging and early-generation direct brain neuroimaging (nuclear brain scanning, angiography, and CT scanning) in the 1970s, to the more precise direct neuroimaging modalities of refined CT scanning and magnetic resonance imaging in the 1980s, the evolution of neurodiagnostic imaging technology has contributed greatly. The fact that three quarters of synchronous lesions occur in patients with lung cancer and that the incidence of lung cancer increased $134 \%$ between 1973 and 1992 also likely contributes to this marked rise in the incidence of synchronous brain metastases. ${ }^{14} \mathrm{~A}$ third explanation for the increased incidence may lie in greater physician and patient awareness of the prognostic importance of diagnosing brain metastases. This awareness results from the knowledge that brain metastases can limit the length and quality of survival in those patients

TABLE 4

Survival analysis summary

\begin{tabular}{lrl}
\hline \hline Characteristic & $\begin{array}{c}\text { No. of } \\
\text { Cases }\end{array}$ & $\begin{array}{c}\text { Median } \\
\text { Survival in } \\
\text { Mos }(95 \% \text { CI })\end{array}$ \\
\hline total no. of cases & 2682 & $3.2(3.0-3.5)$ \\
primary lung/bronchus site & 2023 & $3.3(3.1-3.6)$ \\
primary unknown site & 288 & $3.2(2.5-4.1)$ \\
age group in years & & \\
$0-9$ & 9 & $11.9(0.0-45.9)$ \\
$10-19$ & 10 & $12.2(6.4-24.1)$ \\
$20-29$ & 10 & $56.8(11.2-$ unattained) \\
$30-39$ & 52 & $4.3(3.2-9.1)$ \\
$40-49$ & 262 & $5.2(4.6-6.8)$ \\
$50-59$ & 671 & $4.6(4.1-5.2)$ \\
$60-69$ & 910 & $3.2(3.0-3.7)$ \\
$70-79$ & 605 & $1.7(1.5-2.1)$ \\
$80-89$ & 140 & $1.1(0.7-1.5)$ \\
$90-99$ & 11 & $2.3(0.8-3.5)$ \\
\hline
\end{tabular}


who may otherwise experience prolonged survival after treatment of their primary cancer and that for whom innovative surgical, radiosurgical, and other means have improved outcomes of the treatment of brain metastases.

The definition of "synchronous" varies from study to study, and most authors cite a period of 1 year after the diagnosis of the primary tumor. We defined synchronous as a period of 2 months after diagnosis of a primary tumor to select those patients whose tumor might be considered more biologically "aggressive," but the primary reason for the 2-month period was based on logistical concerns relative to the SEER registry process.

We did not find a correlation between the incidence of synchronous brain metastases and marital status or race. We did not study changes in percentages of diagnosis of metastatic disease based on race or sex over time. Most of these primary lesions were either of poorly or undifferentiated grades. There were no instances of in situ primary cancer associated with brain metastases, a fact that supports the importance of early diagnosis of primary cancer in preventing brain metastases.

Synchronous brain metastases have been associated with a poorer prognosis than metachronous lesions, particularly in cases of lung cancer, ${ }^{1}$ and the survival of patients with metachronous brain metastases has generally ranged from 9 to 14 months $s^{1,5,12,16}$ in selected groups reported in hospital-based studies.

In our study, in patients in the age 20 to 29 -year group the best prognosis was observed; however, survival data based on age must be carefully interpreted because although the best survival data were noted in this group, the number of patients (10) did not allow for a precise estimation. Primary lesions in this group were located as follows: lung (one case), skin (two cases), testicle (two cases), pineal gland (one case), lymph node (one case), and unknown primary (three cases). A more meaningful number of patients was found in the age 55 to 75 -year group who accounted for $65 \%$ of the total group; howev$\mathrm{er}$, the patients in this population experienced a generally poor median survival length of approximately 3.2 months. Although management strategies are not reflected in the overall survival data, it is safe to assume that our current methods of treatment for the primary cancers are clearly ineffective in most patients. Even the understandably selected surgical series show a trend of poorer survival in patients who presented with synchronous lesions. ${ }^{1,5,12,16}$ The overall survival length of the group of 3.2 months reflected the survival of the largest component in this group-primary lung cancer-but survival of patients presently with an unknown primary cancer was also 3.2 months.

To our knowledge, this is the first population-based epidemiological study to report the incidence of synchronous brain metastases and the survival of these patients in North America since 1972. ${ }^{19}$ The poor survival noted in patients with unknown primary cancers is somewhat surprising based on previous reports; however, the results obtained in at least one hospital-based study corroborate this finding. ${ }^{10}$ The reasons for the prolonged survival in a small number of patients cannot be explained based on these data, but the fact that certain patients do experience long-term survival is encouraging and provides a window through which to view future research. Although the predominant prognosis for patients with synchronous metastases is poor, it can be argued that there are individuals for whom treatment may be very beneficial.

There are several limitations of the study. A large number of patients with brain metastases are not diagnosed clinically. Although autopsy studies show approximately a $25 \%$ overall prevalence of brain metastases in patients with systemic cancer, reports on the incidence of metastatic lesion occurring in North America place the risk at 11.1 per 100,000 patients. ${ }^{14,20}$ Based on the current incidence of cancer in general, therefore, one would expect a much higher number of brain metastases..$^{21}$ This smaller number may reflect the fact that patients die before their brain metastases manifest clinically or the clinical impact from other sites of cancer overshadows the neurological symptoms. Thus, even with newer modalities of diagnosis, these data most likely represent an underestimation of the true incidence.

Because the SEER Program does not obtain complete treatment data in patients with metastatic lesions, the effect of treatment on these lesions is difficult to evaluate. There could be favorable subgroups that have responded well to treatment.

\section{References}

1. Andrews RJ, Gluck DS, Konchingeri RH: Surgical resection of brain metastases from lung cancer. Acta Neurochir 138: 382-389, 1996

2. Arseni C, Constantinescu AI: Considerations on the metastatic tumours of the brain with reference to statistics of 1217 cases. Schweiz Arch Neurol Psychiatr 117:179-195, 1975

3. Auchter RM, Lamond JP, Alexander E, et al: A multiinstitutional outcome and prognostic factor analysis of radiosurgery for resectable single brain metastasis. Int J Radiat Oncol Biol Phys 35:27-35, 1996

4. Bentson JR, Steckel RJ, Kagan AR: Diagnostic imaging in clinical cancer management: brain metastases. Invest Radiol 23: 335-341, 1988

5. Burt M, Wronski M, Arbit E, et al: Resection of brain metastases from non-small cell lung carcinoma. Results of therapy. J Thorac Cardiovasc Surg 103:399-441, 1992

6. Caplan LR: Handling of single brain metastasis. Eur Neurol 35:118-126, 1995

7. Cherryman G, Golfieri R: Comparison of spin echo $\mathrm{T}_{1}$-weighted and 90 degrees gadolinium-enhanced magnetic resonance imaging in the detection of cerebral metastases. Br J Radiol 63:712-715, 1990

8. Fogelholm R, Uutela T, Murros K: Epidemiology of central nervous system neoplasms. A regional survey in Central Finland. Acta Neurol Scand 69:129-136,1984

9. Guomundsson KR: A survey of tumors of the central nervous system in Iceland during the 10-year period 1954-1963. Acta Neurol Scand 46:538-552, 1970

10. Hamann G, Meier T, Schimrigk K: [Brain metastases as an initial manifestation of tumor disease.] Nervenarzt 64:104-107, 1993 (Ger)

11. Johnson JD, Young B: Demographics of brain metastases. Neurosurg Clin North Am 7:337-344, 1996

12. Mandell L, Hilaris B, Sullivan M, et al: The treatment of single brain metastasis from non-oat cell lung carcinoma. Cancer 58: 641-649, 1986

13. Mayer RJ, Berkowitz RS, Griffiths CT: Central nervous system involvement by ovarian carcinoma: a complication of pro- 


\section{Epidemiology of synchronous brain metastases}

longed survival with metastatic disease. Cancer 41:776-783, 1978

14. Miller BA, Ries LAG, Hankey BF, et al (eds): SEER Cancer Statistics Review: 1973-1990. NIH Publication No. 93-2729. Bethesda, MD: National Cancer Institute, 1993

15. Mintz AH, Kestle J, Rathbone MP, et al: A randomized trial to assess the efficacy of surgery in addition to radiotherapy in patients with a single cerebral metastasis. Cancer 78:1470-1476, 1996

16. Nakagawa H, Miyawaki Y, Fujita T, et al: Surgical treatment of brain metastases of lung cancer: a retrospective analysis of 89 cases. J Neurol Neurosurg Psychiatry 57:950-956, 1994

17. Newlands ES: Chemotherapy for brain metastases. Prog Exp Tumor Res 29:167-176, 1985

18. Paterson AH, Agarwal M, Lees A, et al: Brain metastases in breast cancer patients receiving adjuvant chemotherapy. Cancer 49:651-654, 1982
19. Percy AK, Elveback LR, Okazaki H, et al: Neoplasms of the central nervous system: epidemiologic considerations. Neurology 22:40-48, 1972

20. Posner JB, Chernik NL: Intracranial metastases from systemic cancer. Adv Neurol 19:579-592, 1978

21. Wingo PA, Tong T, Bolden S: Cancer statistics, 1995. CA Cancer J Clin 45:8-30, 1995

Manuscript received March 27, 2000

Accepted in final form July 10, 2000.

Partial support for this study was provided by National Cancer Institute Contract No. NO1-CN-65064.

Address reprint requests to: Jack P. Rock, M.D., K-11 Neurosurgery, Henry Ford Hospital, 2799 West Grand Blvd., Detroit, Michigan 48202. 